\title{
Non-linear dynamo waves in an incompressible medium when the turbulence dissipative coefficients depend on temperature
}

\author{
A. D. Pataraya $^{1}$, T. A. Pataraya ${ }^{2}$ \\ 1 Abastumany Astrophysical Observatory, Kazbegi Str. 2, 380060, Tbilisi, Republic of Georgia \\ 2 Tbilisi State University, Tbilisi, 380028, Republic of Georgia
}

Received: 7 November 1995/Revised: 19 April 1996/Accepted: 6 May 1996

\begin{abstract}
Non-linear $\alpha-\omega$ dynamo waves existing in an incompressible medium with the turbulence dissipative coefficients depending on temperature are studied in this paper. We investigate of $\alpha-\omega$ solar non-linear dynamo waves when only the first harmonics of magnetic induction components are included. If we ignore the second harmonics in the non-linear equation, the turbulent magnetic diffusion coefficient increases together with the temperature, the coefficient of turbulent viscosity decreases, and for an interval of time the value of dynamo number is greater than 1 . In these conditions a stationary solution of the non-linear equation for the dynamo wave's amplitude exists; meaning that the magnetic field is sufficiently excited. The amplitude of the dynamo waves oscillates and becomes stationary. Using these results we can explain the existence of Maunder's minimum.
\end{abstract}

\section{Introduction}

It is known (Parker, 1979; Priest, 1982) that for excited $\alpha-\omega$ dynamo waves it is necessary to take into account the coefficient of turbulent magnetic diffusion, $\eta$, which is of the order of $\eta=v L$, where $L$ is the moving length. This magnitude is equal to the local scale of altitude for the solar convection zone and is proportional to temperature. Accordingly, we can assume that $\eta=\eta_{0}\left(T / T_{0}\right)^{n_{1}}$, where $T$ and $T_{0}$ are the temperatures of the excited and unexcited medium, respectively. Analogously, we can assume that for the kinematic viscosity $v=v_{0}\left(T / T_{0}\right)^{n_{2}}$, for the coefficient of temperature conductivity $\chi=\chi_{0}\left(T / T_{0}\right)^{n_{3}}$ and that $\alpha=\alpha_{0}\left(T / T_{0}\right)^{n_{4}}$. In the case that the plasma is entirely non-inductive and is not turbulent we have $n_{1}=-1.5$ and $n_{2}=2.5$. Calculations are made in the local Cartesian coordinate system with origin at the centre of the sun. In the second section the non-linear equations for dynamo waves are given, and in the third the adopted results for the sun are discussed.

\section{Investigation of the non-linear dynamo-wave equations}

We investigate dynamo waves with the help of magnetohydrodynamic equations given in the present paper. We assume that the medium is uncompressed and conductive, that the turbulence is dissipative and that $\alpha$ coefficients are dependent on temperature. As mentioned, we use a local Cartesian orthogonal coordinate system originating at the centre of the sun, with the axis $z$ directed locally orthogonal to the solar surface, $y$ directed towards the North pole, locally placed along the tangent of the meridian, and the $x$-axis direction along the west (toroidally).

The main equation of induction is given by (Priest, 1982):

$\partial \boldsymbol{B} / \partial t+(\boldsymbol{v} \boldsymbol{\nabla}) \boldsymbol{B}=(\boldsymbol{B} \boldsymbol{\nabla}) \boldsymbol{v}-\operatorname{rot}(\eta \operatorname{rot} \boldsymbol{B})+\operatorname{rot}\left(\alpha \boldsymbol{B}_{x} \boldsymbol{i}_{x}\right) ;$

the second is the equation of continuity:

$\operatorname{div} \boldsymbol{v}=0, \quad \rho=$ const;

the next is the equation of motion:

$\frac{\partial \operatorname{rot} \boldsymbol{v}}{\partial t}+\operatorname{rot}[\operatorname{rot} \boldsymbol{v}, \boldsymbol{v}]=1 / 4 \pi \rho(\operatorname{rot}[\operatorname{rot} \boldsymbol{B}, \boldsymbol{B}]+4 \pi \operatorname{rot} \boldsymbol{F}) ;$

and the last is an equation of energy:

$$
\begin{aligned}
\partial p / \partial t+(\boldsymbol{v} \nabla) p= & 1 / 4 \pi(\gamma-1) \eta(\operatorname{rot} \boldsymbol{B})^{2} \\
& +(\gamma-1) \operatorname{div}(\chi \nabla p)+(\gamma-1) H .
\end{aligned}
$$

Here $\boldsymbol{B}$ and $\boldsymbol{v}$ are the vectors of magnetic induction and velocity, respectively, $\rho=$ density, $p=$ pressure, $\gamma$ is relative heat, $\boldsymbol{i}_{x}$ is the unit vector along the $x$-axis and $B_{x}=x$ component of magnetic induction. Finally, we have the equation of ideal gaze $p=(\mathrm{R} / \mu) \rho T$, where $T=$ temperature, $\mathrm{R}=$ Gasevus constant, $\mu=$ average atomic mass and $\chi$ is a coefficient of thermal conductivity. 
In Eqs. 3 and 4, $\boldsymbol{F}$ and $H$ are the forces of viscosity and effective viscous dissipation, respectively. From Priest (1982) we consider:

$F_{i}=\rho \sum_{k=1}^{3} \frac{\partial}{\partial x_{k}}\left(v \frac{\partial v_{i}}{\partial x_{k}}\right)$,

$H=\rho v \frac{1}{2} \sum_{i, k=1}^{3}\left(\frac{\partial v_{i}}{\partial x_{k}}+\frac{\partial v_{k}}{\partial x_{i}}\right)^{2}$,

where $x_{1}=x, x_{2}=y, x_{3}=z, v_{1}=v_{x}, v_{2}=v_{y}, v_{3}=v_{z}$. The coefficients $\eta, v, \chi$ and $\gamma$ are dependent on temperature:

$\eta=\eta_{0}\left(T / T_{0}\right)^{n_{1}}$

$v=v_{0}\left(T / T_{0}\right)^{n_{2}}, \quad \chi=\chi_{0}\left(T / T_{0}\right)^{n_{3}}$,

$\alpha=\alpha_{0}\left(T / T_{0}\right)^{n_{4}}$.

Here, $\eta_{0}, v_{0}, \chi_{0}$ and $\alpha_{0}$ are constant values.

Equations 1-4 can be solved with the help of perturbation theory. All the functions take the following form: $f=f_{0}+f_{1}^{\prime}$; where $f_{0}$ is an unperturbed term and $f_{1}^{\prime}$ is perturbed function. Let us consider that the unperturbed density $\rho=\rho_{0}$, pressure $=p_{0}$, the unperturbed quantity of the magnetic field is equal to zero and $v_{0}$ (velocity) has only $x$ components. Then,

$v_{0}=\left(v_{x 0}+v_{x y} y+v_{x z} z\right) \boldsymbol{i}_{x}$,

where $v_{x 0}, v_{x y}$, and $v_{x z}$ are constant quantities.

In accordance with Eq. 4 we investigate the non-perturbed state (gravity is ignored):

$\partial p_{0} / \partial t+\left(\boldsymbol{v}_{0} \boldsymbol{\nabla}\right) p_{0}=\operatorname{div}\left(\chi_{0} \boldsymbol{\nabla} p_{0}\right)+(\gamma-1) H_{0}$,

where

$H_{0}=\rho_{0} v_{0}\left(v_{x y}^{2}+v_{x z}^{2}\right)$.

We estimate the characteristic length $L$ and time $t_{x}$ when the non-perturbed pressure is changed. The estimation is made in the convected region, where $p_{0}=8 \times 10^{11} \mathrm{din} \mathrm{sm}^{-1}$, $\rho_{0}=10^{-2} \mathrm{~g} \mathrm{sm}^{-3}, \chi \hat{=} v_{0}=10^{12} \mathrm{sm}^{2} \mathrm{~s}^{-1},\left|V_{X Y}\right| \hat{=}\left|V_{X Z}\right| \hat{=} \Omega$, $\Omega$ is frequency of the sun rotation, $\left|V_{X Y}\right| R_{Q} \leq 10^{10} \mathrm{sm} \mathrm{s}^{-1}$, and $R_{Q}$ is the radius of the sun.

First we determine $L$; if $\partial p_{0} / \partial t=0$ then $\left|\nabla p_{0}\right| \hat{=} p_{0} / L$. In accordance with Eq. 11 we obtain: $L=10^{6} R_{Q}$. When $\left(\boldsymbol{\nabla} p_{0}\right)=0$ and $\partial p_{0} / \partial t \hat{=} p_{0} / t_{x}$, then $t_{x} \hat{=} 10^{5} t_{0}\left(t_{0}=\right.$ year 22$)$. In these conditions we can suggest that $p_{0}$ depends weakly on time and position. In this case we can use $p_{0}$ as a constant in the equations for perturbed quantities.

For the perturbed terms of Eqs. 1-4 we obtain:

$$
\begin{aligned}
\partial \boldsymbol{B}^{\prime} / \partial t+\left(\boldsymbol{v}_{0} \boldsymbol{\nabla}\right) \boldsymbol{B}^{\prime}= & -\left(\boldsymbol{v}^{\prime} \boldsymbol{\nabla}\right) \boldsymbol{B}^{\prime}+\left(\boldsymbol{B}^{\prime} \boldsymbol{\nabla}\right) \boldsymbol{v}^{\prime}+\eta_{0} \operatorname{rot} \\
& \times\left[\left(1+T^{\prime} / T_{0}\right)^{n_{1}} \operatorname{rot} \boldsymbol{B}^{\prime}\right] \\
& +\operatorname{rot}\left[\alpha_{0}\left(1+T^{\prime} / T_{0}\right)^{n_{4}} B_{x} \boldsymbol{i}_{x}\right]
\end{aligned}
$$

$\partial\left(\operatorname{rot} \boldsymbol{v}^{\prime}\right) / \partial t+\left(\boldsymbol{v}_{0} \boldsymbol{\nabla}\right) \operatorname{rot} \boldsymbol{v}^{\prime}=\left(\operatorname{rot} \boldsymbol{v}_{0} \boldsymbol{\nabla}\right) \boldsymbol{v}^{\prime}+\left(\operatorname{rot} \boldsymbol{v}^{\prime} \boldsymbol{\nabla}\right) \boldsymbol{v}_{0}$

$$
\begin{aligned}
& +\left(\operatorname{rot} \boldsymbol{v}^{\prime} \boldsymbol{\nabla}\right) \boldsymbol{v}^{\prime}-\left(\boldsymbol{v}^{\prime} \boldsymbol{\nabla}\right) \operatorname{rot} \boldsymbol{v}^{\prime} \\
& +\operatorname{rot}\left[\operatorname{rot} \boldsymbol{B}^{\prime}, \boldsymbol{B}^{\prime}\right] / 4 \pi \rho+\operatorname{rot} \boldsymbol{F}^{\prime} / \rho ;
\end{aligned}
$$

$$
\begin{aligned}
\partial p^{\prime} / \partial t+\left(\boldsymbol{v}_{0} \boldsymbol{\nabla}\right) p^{\prime}= & -\left(\boldsymbol{v}^{\prime} \boldsymbol{\nabla}\right) p^{\prime}+(\gamma-1) \eta_{0}\left(1+T^{\prime} / T_{0}\right)^{n_{1}} \\
& \times\left(\operatorname{rot} \boldsymbol{B}^{\prime}\right)^{2} / 4 \pi+\chi_{0} \operatorname{div}\left[1+T^{\prime} / T_{0}\right)^{n_{3}} \\
& \left.\times \boldsymbol{\nabla}\left(T^{\prime} / T_{0}\right)\right]+(\gamma-1)\left(H-H_{0}\right) .
\end{aligned}
$$

These equations are correct only for $t^{\prime}$ and $L$ satisfying the following conditions: $t^{\prime} \ll 10^{5} t_{0}\left(t_{0}=\right.$ year 22$), L \ll 10^{6} R_{Q}$.

The perturbation terms in Eqs. 13-15 we can consider as $\left(\rho^{\prime}=0\right)$ :

$$
\begin{aligned}
& B_{x}=B_{0} u_{2} \exp (\imath \varphi)+C . C ., \\
& B_{y}^{\prime}=B_{0} \Delta^{-1} k_{z} \omega u_{4} \exp (\imath \varphi)+C . C ., \\
& B_{z}^{\prime}=-B_{0} \Delta^{-1} k_{y} \omega u_{4} \exp (\imath \varphi)+C . C ., \\
& T / T_{0}=u_{0}+u_{6} \exp (\imath 2 \varphi)+u_{6}^{*} \exp (-\imath 2 \varphi), \\
& V_{x}^{\prime}=u_{8} \exp (\imath 2 \varphi)+C . C ., \\
& V_{y}^{\prime}=u_{10} \exp (\imath 2 \varphi)+C . C ., \\
& V_{z}^{\prime}=u_{12} \exp (\imath 2 \varphi)+C . C ., \\
& \varphi=k_{y} y+k_{z} z+\delta \omega \int_{0}^{\tau}\left(1+u_{0}\right)^{n_{4} / 2} d t
\end{aligned}
$$

Here, $k_{y}$ and $k_{z}$ are the $y$ and $z$ components of the wave number, $\omega=\sqrt{\left|\alpha_{0} \Delta\right| / 2}$ is the frequency of the linear dynamo waves, $\Delta=k_{z} v_{x y}-k_{y} v_{x z}, \delta=1$ when $\alpha_{0} \Delta>0$ and -1 when $\alpha_{0} \Delta<0, C$.C. stands for complex conjuction; $B_{0}$ is constant, determined as a value of perturbed magnetic induction at $t=0 ; u_{0}$ is a real function of the variable $\tau$, i.e. $\operatorname{Im} u_{0}=0$, and in Eq. $19 u_{6}^{*}$ denotes the complex conjuction of the function $u_{6}$.

The components of magnetic induction include the characteristic phase of oscillation $\varphi$, which, as we can see from Eq. 23, depends on $\omega$. Taking into account these conditions, we can suggest that the perturbed magneticinduction oscillations include only the first harmonics of the dynamo waves. According to Eq. 19, perturbed pressure consists of the terms with zero and second harmonics of dynamo waves, but the perturbed velocity components from Eqs. 21-23 contain the second harmonics of the dynamo waves only. We can also consider that in Eqs. 13-15 the terms of higher than second-order harmonics are negligibly small and are ignored.

As we can see from Eqs. 16-18, $B_{0} u_{2}$ is an amplitude of the $x$-component of magnetic induction, and $B_{0} u_{4}$ is an amplitude of the function $\left(\boldsymbol{B}^{\prime} \boldsymbol{V}\right) v_{x} / \omega$. We should mention that all the perturbed values are homogeneous in the $x$ direction; for Eqs. $13-15, \delta / \delta x=0$.

We investigate dynamo waves without taking into account the second harmonics of dynamo waves for the perturbed velocity and pressure. In this case the following equations are adopted:

$d u_{0} / d \tau=C_{1} L_{1}\left(\left|u_{2}\right|^{2}+d_{0}\left|u_{4}\right|^{2}\right)+p_{m} C_{2}\left(L_{2}-N^{-1 / 2}\right)$,

$d u_{2} / d \tau=u_{4}-\left[L_{1}+i \delta\left(1+u_{0}\right)^{n_{4} / 2}\right] u_{2}$,

$d u_{4} / d \tau=i 2 \delta\left(1+u_{0}\right)^{n_{4}} u_{2}-\left[L_{1}+i \delta\left(1+u_{0}\right)^{n_{4} / 2}\right] u_{4}$,

where $\tau=\omega t, \quad C_{1}=2(\gamma-1) \beta^{-1}, \quad C_{2}=\gamma(\gamma-1) M_{T 1}^{2}$, $\beta=4 \pi p_{0} B_{0}^{-2}, M_{T 1}=V_{1} V_{T}^{-1}, V_{1}=K^{-1}\left(V_{x y}^{2}+V_{x z}^{2}\right)^{1 / 2}$, 
$V_{T}=\left(\gamma p_{0} \rho_{0}^{-1}\right)^{1 / 2}$ is the sound velocity in the non-perturbed medium, $p_{m}=v_{0} \eta_{0}^{-1}$ is the Prandtl magnetic number (Priest, 1982), $\quad d_{0}=\omega^{2} k^{2} \Delta^{-2}, \quad k^{2}=k_{z}^{2}+k_{y}^{2}, \quad L_{1}=$ $\left(1+u_{0}\right)^{n_{1}} N^{-1 / 2}, L_{2}=\left(1+u_{0}\right)^{n_{2}} N^{-1 / 2}$ and $N=\omega^{2} \eta^{-2} k^{4}$ is the dynamo number (Priest, 1982).

We now turn to Eqs. 24-26 with $n_{2}=0$ and $n_{4}=0$, and with the following origin conditions: when $\tau=0$, $\operatorname{Re} u_{2}=1, \operatorname{Im} u_{2}=0, \operatorname{Re} u_{4}=1, \operatorname{Im} u_{4}=\delta$ and $u_{0}=0$. Using all these conditions we obtain:

$u_{2}=\left[\Phi\left(u_{0}\right)\right]^{1 / 2}$,

$u_{4}=(1+i \delta)\left[\Phi\left(u_{0}\right)\right]^{1 / 2}$,

$\tau=C_{10}^{-1} N^{1 / 2} \int_{0}^{u_{0}}(1+\xi)^{-n_{1}}[\Phi(\xi)]^{-1} d \xi$,

$\Phi\left(u_{0}\right)=1+2 C_{10}^{-1}\left\{N^{1 / 2}\left(1-n_{1}\right)^{-1}\left[\left(1+u_{0}\right)^{1-n_{1}}-1\right]-u_{0}\right\}$,

where $C_{10}=C_{1}\left(1+2 d_{0}\right), N>1$ and $u_{0}>0$. We have to find the moment of the time $t_{1},\left(\tau_{1}=\omega t_{1}\right)$, when $u_{2}$ reaches its maximal value $\left(u_{2}\right)_{1}=u_{2 \max }$, and $\left(u_{0}\right)_{1}=u_{01}$. At the moment $\left(L_{1}\right)_{1}=L_{10}=1$,

$\left.\frac{d^{2} u_{2}}{d \tau^{2}}\right|_{r=r_{1}}=-2 n_{1} N^{-1 / 2 n_{1}}\left[\Phi\left(u_{01}\right)\right]^{1 / 2}$,

$t_{1}=\omega^{-1} C_{10}^{-1} N^{1 / 2} \int_{0}^{u_{01}}(1+\xi)^{-n_{1}}[\Phi(\xi)]^{-1} d \xi$,

where $u_{01}=N^{1 /\left(2 n_{1}\right)}-1$.

In accordance with Eq. 31, $u_{2}$ reaches its maximal value when $n_{1}>0$. From Eqs. 27 and 29, when $u_{2}=1$ we can consider that the moment of time $t_{2}>0\left(t_{2}=\omega t_{2}\right)$ can be given as:

$t_{2}=\omega^{-1} C_{10}^{-1} N^{1 / 2} \int_{0}^{u_{02}}(1+\xi)^{-n_{1}}[\Phi(\xi)]^{-1} d \xi$.

Here $u_{02}$ is a solution of the following algebraic equation:

$N^{1 / 2}\left(1-n_{1}\right)^{-1}\left[\left(1+u_{02}\right)^{1-n_{1}}-1\right]-u_{02}=0$.

When $n_{1}=1$, Eq. 34 can be given as:

$N^{1 / 2} \ln \left(1+u_{02}\right)-u_{02}=0$,

with

$n_{1}=2, \quad u_{02}=N^{1 / 2}-1$.

Investigating Eqs. $27-33$ when $N \approx 1$, we obtain:

$u_{2 \max }=\left(1+\alpha_{n}\right)^{1 / 2}$,

$t_{1}=\left[n_{1} C_{10} \omega^{2}\left(1+\alpha_{n}\right)\right]^{-1 / 2} \ln \left[\left(1+\alpha_{n}\right)^{1 / 2}+\alpha_{n}{ }^{1 / 2}\right]$,

$t_{2} \approx 2 t_{1}$.

Here $\alpha_{n}=(N-1)^{2} /\left(4 n_{1} C_{10}\right), n_{1}>0$ and $N>1$.

Now we investigate the stationary solution of Eqs. 24-26. We mark the stationary value of the functions as: $u_{2}=u_{20}, u_{4}=u_{40}, u_{0}=u_{00}$, thus obtaining

$u_{40}=(1+i \delta) u_{20}\left(1+u_{00}\right)^{n_{4} / 2}$,

$u_{00}=-1+N^{1 /\left(2 n_{1}-n_{4}\right)}$,

$C_{1}\left(1+u_{00}\right)_{4}^{n / 2}\left[1+2 d_{0}\left(1+u_{00}\right)_{4}^{n}\right]\left|u_{20}\right|^{2}$

$=\mathrm{PmC}_{2} N^{-1 / 2}\left[1-\left(1+u_{00}\right)^{n_{2}}\right]$.
In accordance with Eqs. 41-42, for the existence of the stationary solutions it is necessary to satisfy the following inequality:

$1>N^{n_{2} /\left(2 n_{1}-n_{4}\right)}$.

This can be satisfied by one or other of the following conditions:

$N>1, \quad n_{2} /\left(2 n_{1}-n_{4}\right)<0$,

or

$N<1, \quad n_{2} /\left(2 n_{1}-n_{4}\right)>0$.

We investigate the stationary state of the solution of Eqs. 24-26 with the help of the perturbation theory for non-linear waves. If the perturbed value is proportional to $\exp (q \tau)$, we get a fourth-order algebraic equation. The discussion of this equation shows us that $\operatorname{Re} q<0$ (Korn and Korn, 1968) if we satisfy the inequalities.

$n_{2}<0$,

$2 n_{1}-n_{4}>0$

so that $n_{2} /\left(2 n_{1}-n_{4}\right)<0$ and, in accordance with Eq. 44 , $N>1$.

When $n_{4}=0$, the criterea of stability have the following form:

$n_{2}<0, \quad n_{1}>0,\left(1+\left|n_{2}\right| / n_{1}\right)^{\left(2 n_{1}-n_{4}\right) /\left|n_{2}\right|}>N>1$.

\section{Discussion}

When the coefficients of viscosity and $\alpha$ are considered to be constant values $\left(n_{2}=0, n_{4}=0\right)$, the asymptotic solutions for the amplitudes of the dynamo waves are given in Eqs. 27-30. It is shown that the amplitude of the magnetic field reaches its maximal value (the magnetic field is at its strongest) when $n_{1}>0 ; t$ is the period of time during which this maximal value is reached (Eq. 39). The equation of the dependence of the magnetic field on the dynamo number is obtained. We can say exactly, that the magnetic field is strengthened when the dynamo number $N>1$. In the case $N \approx 1$, the rate of strengthening of the magnetic field (ratio of the amplitude of the magnetic field to its meaning when $t=0)$ is proportional to $(N-1)^{2} \beta_{0}$, where $\beta_{0}=4 \pi p_{0} / B_{0}^{2}$ for the non-perturbed medium. We can see that the strengthening of the magnetic field is strong when $(N-1)^{2} \beta_{0} \gg 1$.

In the case $n_{1} \neq 0, n_{2} \neq 0$ and $n_{4} \neq 0$, the stationary solutions of the equation for the amplitude of the magnetic field and pressure are given. The conditions of stability of this solution have the following form $(\operatorname{Re} q<0)$ :

$n_{1}>0, n_{2}<0,2 n_{1}-n_{4}>0$ and $N_{1}>N>1$;

here $N_{1}=\left(1+\left|n_{2}\right| n_{1}^{-1}\right)^{\left(2 n_{1}-n_{4}\right) /\left|n_{2}\right|}$.

If $N<N_{1}$ and $|\operatorname{Re} q|>|\operatorname{Im} q|$, the perturbed amplitude quickly decreases and, oscillating, approaches a stationary state. The period of oscillation lasts several hundred years, 
it compares on the values of the Dynamo number, $p_{m}$ and $\beta_{0}$. It is seen, therefore, that oscillations account for the existence of the Maunder minimum.

Acknowledgements. We would like to thank the Soros Foundation for the financial support necessary for our theoretical research.

Topical Editor R. Schwenn thanks one referee for his help in evaluating this paper.

\section{References}

Korn, G. A., T. M. Korn, Mathematical Handbook, McGraw-Hill, New York, Hamburg, 1968.

Parker, E. N., Cosmical Magnetic Fields, Moscow, Clarendon Press, Oxford, 1979.

Priest, E. R., Solar Magnetohydrodynamics, D. Reidel, Dordrecht, 1982. 